\title{
COVID-19 infection in kidney transplant recipients: report from two centers of Bangladesh
} \author{
Rahman $^{2}$, Harun Ur Rashid ${ }^{1}$ \\ ${ }^{1}$ Department of Nephrology and Transplantation, Kidney Foundation Hospital and Research Institute, Dhaka, Bangladesh
${ }^{2}$ Department of Nephrology, Urology and Transplantation, Center for Kidney Diseases and Urology, Dhaka, Bangladesh
}

Nura Afza Salma Begum¹, Tasnuva Sarah Kashem', Farnaz Nobi Rima', Shakib Uz-Zaman Arefin', Kamrul Islam², Rezwanur

Background: The novel coronavirus severe acute respiratory syndrome coronavirus 2 (SARS-CoV-2) had affected people around the globe including transplant recipients. Here we report our experience of coronavirus 2019 (COVID-19) infection in kidney transplant recipients of Bangladesh.

Methods: This prospective observational study was performed in two specialized kidney hospitals of Dhaka, Bangladesh from May 2020 to August 2020.

Results: In these two centers about 1,043 kidney transplant recipients were regularly followed up. Among them 31 patients (2.97\%) developed COVID-19 infection during the study period. Of them 23 patients were test positive and the remaining test negative but suspected clinically and radiologically. Average age of recipients was $38.3 \pm 6.9$ years $(23-53)$. Twenty-eight recipients were male $(90.3 \%)$ and three were female $(9.7 \%)$. Fever was the most common presentation $(100 \%)$, followed by cough $(80.6 \%)$, shortness of breath (35.5\%), diarrhoea (12.9\%), generalized weakness (12.9\%), loss of taste $(9.7 \%)$, headache $(6.5 \%)$, body ache $(6.5 \%)$, throat pain (3.2\%), chest pain (3.2\%), abdominal pain (3.2\%), and orbital cellulitis (3.2\%). On investigation, leukocyte count was normal in $77 \%$ cases and raised in $19.2 \%$ cases and lymphopenia was observed in $69.2 \%$ cases. C-reactive protein (CRP) was found high in $63.6 \%$ recipients. Chest X-ray showed pneumonic changes in $80.6 \%(25 / 31)$ cases. Flavipiravir was given to 20 transplant recipients $(64.5 \%)$. Five patients (16.1\%) developed acute kidney injury (AKI); one patient improved and four patients $(80 \%)$ had died, three patients required dialysis. The overall mortality rate was $22.6 \%$ (7 out of 31 ); three patients had severe AKI (42.8\%), two developed acute respiratory distress syndrome (28.6\%) and two developed cerebrovascular disease (28.6\%).

Conclusions: In summary kidney transplant recipients in our centers had a higher rate of COVID-19 infection and higher rate of mortality than the general population (22.6\% vs. $1.4 \%)$. Patients who developed AKI had a higher mortality rate.

Corresponding author: Nura Afza Salma Begum

E-mail: nuraafzanupur@yahoo.com

\section{() The Korean Society for Transplantation}

This is an Open Access article distributed under the terms of the Creative Commons Attribution Non-Commercial License (http://creativecommons.org/licenses/by-nc/4.0/) which permits unrestricted non-commercial use, distribution, and reproduction in any medium, provided the original work is properly cited. 